\title{
STRETCHING THE CIRCULAR CYLINDRICAL ELASTIC SHEET*
}

\author{
BY \\ B. F. BOWMAN \\ University of Wisconsin and Mobil Exploration Services
}

\begin{abstract}
The theory of thin elastic sheets, neglecting bending, is applied to many thin surface problems. The classical theory for these surfaces is inadequate when the strains are small, the surface is unstressed before deformation, and boundary displacements are prescribed. The equations in this case are degenerate. Attempts to solve the exact equations for these elastic membranes have failed for small strains. The purpose of this paper is to determine the behavior of the solution to the exact equations for the elastic surface which is initially a circular cylinder and which is deformed by a uniform pull at the ends keeping the end radii fixed. A resolution of the small-strain behavior both analytically and numerically is of particular importance.

It was found that the stresses were statically determinant and that the equations could be reduced to a single nonlinear ordinary differential equation with boundary values. This equation could be converted into a nonlinear integral equation. By using this equation it was possible to prove existence and uniqueness and determine the appropriate linear theory for small values of the strains. All results were verified by numerically solving the integral equation using Simpson's rule and the nonlinear Gauss-Seidel method. The truncation error was analyzed. The numerical results were also compared to the Bromberg-Stoker theory (a large displacement theory), and to another exact strain treatment due to Stoker.
\end{abstract}

1. Background. The membrane theory of shells is based on the assumption that bending forces can be neglected. When the membrane is assumed to be unstressed in its undeformed state, the classical linear theory of membranes is inapplicable. This theory results from linearizing the governing equations about a large prestressed state. A straightforward linearization of the governing equations yields a solution incapable of satisfying arbitrary boundary conditions.

One resolution of this problem has been studied. Experiments by Ecke [9] confirmed earlier studies made by Foppl and Hencky which suggested that changes in the normal displacement could be of more significance than tangential changes. Foppl [10] studied this for symmetric deformations of plane circular membranes. In a now-classical paper in this area, Bromberg and Stoker [2] generalized Foppl's theory to arbitrary surfaces of revolution. The change expounded was to retain all nonlinear terms involving derivatives of the normal displacement. As a particular example, Bromberg and Stoker dealt with the spherical cap in some detail. They found that for small strains their equations became singular. This difficulty was overcome by developing an appropriate boundary-layer

\footnotetext{
* Received December 26, 1979. Partial support provided by NSF Grant MPS 75-06366.
} 
analysis. More recently, numerical work by Goldberg [11] substantiates their results for small strains.

This theory was applied to the flat circular sheet and was also studied by Hencky [12]. Some open mathematical questions concerning his results led to further study by Dickey [8] who was able to solve the equations numerically for sufficiently small strains to indicate that in this geometry no boundary layer occurs. This was established analytically by Callegari and Reiss $[3,4]$.

For the circular cylinder, the Bromberg-Stoker equations can be solved exactly. No boundary layer occurs.

Reissner [14] developed a different theory. Here, too, small strains proved difficult. His analysis of the spherical cap gave the same result as that obtained by Bromberg and Stoker.

A resolution of the difficulties posed by the membrane theory when the membrane is initially unstressed lies in determining the correct "linear" approximation (i.e., smallstrain approximation) of the nonlinear membrane equations. Consequently, it is of interest to study the exact equations. Those of the circular cylinder are the least formidable. Stoker [15] tried solving the exact equations of the circular cylinder by a finite difference method, but was unable to get convergence for small strains. Corneliussen and Shield [5] prestrained the cylinder with an inflation and extension to avoid the small strain difficulty. Wu $[18,19]$ solved the equations by quadrature, but did not discuss the case of small strains where difficulties with the integrals and certain transformations could arise.

In this paper another approach is taken. The investigation is addressed to a prototype thin-walled elastic cylinder, in which deformation results from stretching the cylinder so that the ends retain fixed radii and the surface remains a surface of revolution. The deformations considered here allows the further assumption that they depend only upon position along the $x$-axis.

The metric strains, $\varepsilon_{x}$ and $\varepsilon_{\theta}$, are defined by considering the difference $\left(d s^{*}\right)^{2}-(d s)^{2}$, where $d s^{*}$ and $d s$ are the arclengths of a line segment on the surface after and before deformation, respectively. Therefore,

$$
\left(d s^{*}\right)^{2}-(d s)^{2}=2\left[\varepsilon_{x}(d x)^{2}+\varepsilon_{\theta}(a d \theta)^{2}\right] .
$$

For the strain energy functional $V_{1}$ defined by Eq. (1.2), the equilibrium equations are the Euler equations which minimize $V_{1}$ over all admissible displacements $u$ and $w$ :

$$
V_{1}=\frac{\pi h a E}{\left(1-v^{2}\right)} \int_{0}^{L}\left[\varepsilon_{x}^{2}+\varepsilon_{\theta}^{2}+2 v \varepsilon_{x} \varepsilon_{\theta}\right] d x
$$

where $h$ is the thickness of the cylinder wall, $a$ is the undeformed cylinder radius, $2 L$ is the length of the cylinder, $E$ is Young's modulus, $v$ is the Poisson ratio.

The governing system of equations for the cylinder described by $V_{1}$ are listed below, where ' denotes differentiation with respect to $x$ :

$$
\begin{aligned}
\varepsilon_{x} & =u^{\prime}+\frac{1}{2}\left[\left(u^{\prime}\right)^{2}+\left(w^{\prime}\right)^{2}\right], \\
\varepsilon_{\theta} & =-\frac{w}{a}+\frac{1}{2}\left(\frac{w}{a}\right)^{2}, \\
\sigma_{x} & =\frac{E}{\left(1-v^{2}\right)}\left(\varepsilon_{x}+v \varepsilon_{\theta}\right),
\end{aligned}
$$




$$
\begin{gathered}
\sigma_{\theta}=\frac{E}{\left(1-v^{2}\right)}\left(\varepsilon_{\theta}+v \varepsilon_{x}\right), \\
{\left[\left(1+u^{\prime}\right) \sigma_{x}\right]^{\prime}=0,} \\
{\left[\sigma_{x} w^{\prime}\right]^{\prime}=\frac{\sigma_{\theta}}{a}\left(\frac{w}{a}-1\right),} \\
w(L)=0, \quad w^{\prime}(0)=0, \\
u(0)=0, \quad w(0)=\gamma .
\end{gathered}
$$

In the above, $u$ and $w$ are displacements of the middle surface of the cylinder wall; $\sigma_{x}$ and $\sigma_{\theta}$ are "generalized stresses"; $\gamma$ is the contraction at the center.

Before proceeding, however, a few remarks are in order to put this model into perspective with the other models. The strains used in (1.3)-(1.12) were used by Foppl and in the Bromberg-Stoker theory. When all nonlinear terms are dropped in (1.3)-(1.4), they are the strains used in the classical linear theory. In [15] and [18] the strains were defined by $\left(d s^{*} / d s-1\right)$. These are related to the metric strains by

$$
\begin{aligned}
& e_{x}=\sqrt{1+2 \varepsilon_{x}}-1, \\
& e_{\theta}=\sqrt{1+2 \varepsilon_{\theta}}-1,
\end{aligned}
$$

and for small values of $\varepsilon_{x}$ and $\varepsilon_{\theta}$ they should be comparable theories.

The $\sigma_{x}$ and $\sigma_{\theta}$ defined by (1.5) and (1.6) are introduced for convenience only. They are not to be confused with the stresses $\sigma_{x}^{*}$ and $\sigma_{\theta}^{*}$ which are related by

$$
\begin{aligned}
& \sigma_{x}^{*}=\sqrt{\frac{\left(1+2 \varepsilon_{x}\right)}{\left(1+2 \varepsilon_{\theta}\right)}} \sigma_{x}, \\
& \sigma_{\theta}^{*}=\sqrt{\frac{\left(1+2 \varepsilon_{\theta}\right)}{\left(1+2 \varepsilon_{x}\right)}} \sigma_{\theta} .
\end{aligned}
$$

As with the linear extensions, when the strains are small, the "generalized stresses" are essentially the stresses; cf. [13].

The last boundary condition, (1.12), was also used in [15]. In [18], the end displacement was specified.

2. Reduction to a nonlinear integral equation for $\varepsilon_{\theta}$. The system (1.3)-(1.12) can be made nondimensional by scaling as follows:

$$
\begin{gathered}
W=w / a, \quad U=u / a, \\
\Sigma_{x}=\sigma_{x} / E, \quad \Sigma_{\theta}=\sigma_{\theta} / E, \\
s=x / a, \quad B=L / a, \quad \Gamma=\gamma / a .
\end{gathered}
$$

The system (1.3)-(1.12) can be reduced to a single equation for the function $\varepsilon_{\theta}^{\prime}$. (' will now denote differentiation with respect to $s$.) The procedure is to eliminate all functions but $\Sigma_{x}$ and $\varepsilon_{\theta}$. This can be done by integrating (1.7) and using (1.3) to eliminate the quantity $\left(1+u^{\prime}\right)$. Eqs. (1.5) and (1.6) can be solved for $\varepsilon_{x}$ and $\Sigma_{\theta}$. Likewise, assuming for 
now that contraction to a point will not occur for small strains, $W$ can be expressed as a function of $\varepsilon_{\theta}$. These equations are substituted into (1.7) and (1.8). The result is:

$$
\begin{gathered}
{\left[2\left(1-v^{2}\right) \Sigma_{x}-2 v \varepsilon_{\theta}+1-\left(\varepsilon_{\theta}^{\prime} / \sqrt{1+2 \varepsilon_{\theta}}\right)^{2}\right] \Sigma_{x}^{2}=\Lambda^{2},} \\
\left(\Sigma_{x} \varepsilon_{\theta}^{\prime} / \sqrt{1+2 \varepsilon_{\theta}}\right)^{\prime}=\left(\varepsilon_{\theta}+v \Sigma_{x}\right) \sqrt{1+2 \varepsilon_{\theta}} .
\end{gathered}
$$

(The constant of integration $\Lambda$ cannot be determined from the given data.) These equations can be integrated to find an algebraic relationship between $\Sigma_{x}$ and $\varepsilon_{\theta}$ :

$$
3\left(1-v^{2}\right) \Sigma_{x}^{2}+2 \Sigma_{x}-4 v \varepsilon_{\theta} \Sigma_{x}-\varepsilon_{\theta}^{2}=K .
$$

The constant of integration $K$ cannot be determined from the data. Up to this constant, $\Sigma_{x}$ is now known as a function of $\varepsilon_{\theta}$ :

$$
\Sigma_{x}(\varepsilon, K)=\frac{-1+2 v \varepsilon_{\theta}+\left(\left(-1+2 v \varepsilon_{\theta}\right)^{2}+3\left(1-v^{2}\right)\left(\varepsilon_{\theta}^{2}+K\right)\right)^{1 / 2}}{3\left(1-v^{2}\right)} .
$$

Eq. (2.9) can be reformulated more simply by defining a new parameter $\zeta$ by

$$
d \zeta / d s=\left(1+2 \varepsilon_{\theta}\right)^{1 / 2} ; \quad \zeta(0)=0
$$

with

$$
\zeta(B)=\beta, \quad \varepsilon(\zeta)=\varepsilon_{\theta}(s(\zeta))
$$

Eq. (2.9) can now be integrated twice using the boundary conditions

$$
\varepsilon_{\theta}(B)=0, \quad \varepsilon_{\theta}^{\prime}(0)=0 .
$$

Define

$$
\begin{aligned}
H(\varepsilon, K) & =\int_{\varepsilon(\zeta)}^{0} \Sigma_{x}(\bar{\varepsilon}, K) d \bar{\varepsilon}, \\
L\left(\zeta, \zeta^{\prime}\right) & =\begin{array}{ll}
\beta-\zeta, & 0 \leq \zeta^{\prime} \leq \zeta, \\
\beta-\zeta^{\prime}, & \zeta \leq \zeta^{\prime} \leq \beta .
\end{array}
\end{aligned}
$$

The problem to be solved is

$$
H(\varepsilon, K)=\int_{0}^{\beta} L\left(\zeta, \zeta^{\prime}\right) \Sigma_{\theta}(\varepsilon, K) d \zeta^{\prime}
$$

with $K$ and $\beta$ determined by the remaining conditions (2.13), (2.14) and (2.20). Existence and uniqueness can be shown using standard techniques (cf. $[1,16])$ :

$$
\varepsilon_{\theta}(0)=\frac{1}{2} \Gamma^{2}-\Gamma=e .
$$

Once $\varepsilon_{\theta}$ is known the other functions are found by computing:

$$
\begin{aligned}
\varepsilon_{x}= & \left(1-v^{2}\right) \Sigma_{x}-v \varepsilon_{\theta}, \\
\Sigma_{\theta}= & \left\{\varepsilon_{\theta}+v\left[\left(1-v^{2}\right) \Sigma_{x}-v \varepsilon_{\theta}\right]\right\} /\left(1-v^{2}\right)=\varepsilon_{\theta}+v \Sigma_{x}, \\
W= & 1-\sqrt{1+2 \varepsilon_{\theta},} \\
& \left(1+U^{\prime}\right)^{2}=2 \varepsilon_{x}+1-\left(W^{\prime}\right)^{2}, \quad U(0)=0,
\end{aligned}
$$

and $\Sigma_{x}$ from (2.11). 
3. Derivation of the linear equations. If $\varepsilon_{x}$ and $\varepsilon_{\theta}$ are small then (1.5) indicates that $\Sigma_{x}$ will also be small. Define

$$
\Sigma_{x}[\varepsilon(\beta), K]=k .
$$

On this basis, the integral equation (2.19) is expanded for small strains, i.e., small values of $k$ and $\varepsilon$. The procedure is to look for a stretching

$$
\tau=\phi k^{\gamma} ; \quad T=\beta k^{\gamma},
$$

which will remove the degeneracy when strains are small. It is assumed that

$$
\varepsilon(\phi)=\varepsilon(\tau ; k) ; \quad \varepsilon(\tau ; 0)=0 .
$$

The choice $\gamma=-1 / 2$ will preserve the leading terms in the expansions of $\Sigma_{\theta}(\varepsilon, k)$ and $H(\varepsilon, k)$ substituted in (2.19). The equation defined by the coefficient of $k^{2}$ and its solution are

$$
\begin{aligned}
& \varepsilon_{0}(\tau)=\int_{0}^{T} L\left(\tau, \tau^{\prime}\right)\left[\varepsilon\left(\tau^{\prime}\right)+v\right] d \tau^{\prime}, \\
& \varepsilon_{0}(\tau)=-v[1-\cosh (\tau) \operatorname{sech}(T)], \\
& \varepsilon_{0}(\tau)=\frac{\partial \varepsilon}{\partial k}(\tau ; 0) .
\end{aligned}
$$

As $k$ is taken to zero, $\left|\varepsilon-k \varepsilon_{0}\right|$ goes to zero and, hence, $k \varepsilon_{0}(\zeta / k)$ approximates $\varepsilon$ as desired.

The full solution can now be found from $\varepsilon_{0}$ :

$$
\begin{aligned}
s & \simeq \int_{0}^{\zeta} \frac{d \zeta^{\prime}}{\sqrt{1+2 k \varepsilon_{0}}} \simeq \zeta(1+k v)+O\left(k^{3 / 2}\right), \\
\varepsilon_{\theta}^{*}(s) & =-k v\left\{1-\cosh \left[s / k^{1 / 2}(1+v k)\right] \operatorname{sech}\left[B / k^{1 / 2}(1+v k)\right]\right\} ; \\
W^{*}(s) & =-\varepsilon_{\theta}^{*}, \\
\Sigma_{x}^{*}(s) & =k, \\
\Sigma_{\theta}^{*}(s) & =\varepsilon_{\theta}^{*}+v k, \\
\varepsilon_{x}^{*}(s) & =\left(1-v^{2}\right) k-v \varepsilon_{\theta}^{*}, \quad \\
\left(U^{*}\right)^{\prime} & =\varepsilon_{x}^{*}-\frac{1}{2}\left(W^{* \prime}\right)^{2} ; \quad,=d / d s ; \quad U^{*}(0)=0 .
\end{aligned}
$$

When all powers of $k$ greater than 1 are dropped in (3.7)-(3.13), the solution is that of the Bromberg-Stoker theory.

4. Development of solution for large strains. An interesting result obtained using (1.13) and (1.4) (cf. [15, 17]) was the demonstration that a finite cylinder could be contracted to a point by a finite extension. For the material described by (1.3)-(1.12) this is not possible. If $\gamma=a$, the transformation from $W$ to $\varepsilon_{\theta},(2.23)$, is no longer valid. Consequently, (2.9) is the governing equation when this contraction is specified. The point corresponding to $W=1$ and $d W / d s=0$ is a critical point of the equation with two characteristics passing through it. Only one of these also passes through the line $W=0$, 
so if $t \equiv s-B$ is used as a parameter along this curve, one has $\lim _{t \rightarrow t_{0}} W(B+t)=1$ only if $t_{0}$ is infinite (cf. $\left.[6,7]\right)$. Thus, contraction to a point cannot be achieved.

The same result is obtained by examining the integral equation (2.19) for large strains. The limit solution is

$$
\begin{aligned}
\varepsilon_{\infty}(s) & =\frac{-\sinh [\sqrt{ } v(B-s)] \sinh [\sqrt{ } v(B+s)]}{2 \cosh ^{2}[\sqrt{ } v B]}, \\
W(s) & =1-\cosh [\sqrt{ } v s] \operatorname{sech}[\sqrt{ } v B], \\
\Sigma_{x} & =\frac{k \sqrt{f^{*}\left(\varepsilon_{\infty}, \delta\right)}}{3\left(1-v^{2}\right)}+O(1) \approx k, \\
\Sigma_{\theta} & \approx v k \\
\varepsilon_{x} & \approx\left(1-v^{2}\right) k-v \varepsilon_{\infty}(s), \\
\Lambda & \approx \sqrt{2\left(1-v^{2}\right)} k^{3 / 2}, \\
U & \approx \sqrt{2\left(1-v^{2}\right) k} s .
\end{aligned}
$$

This solution shows that as long as $B$ is finite, $W$ will never contract, the conclusion reached earlier from the differential equation. Since in the limit, $\varepsilon_{\infty}$ does not depend on $k$ but rather on $B$, "large strain" may be revised to mean that $\varepsilon_{x}$ is large and $\varepsilon_{\theta}$ is close to its limiting value.

5. Numerical results. Although it was noted that the integral equation retains the singular behavior of the membrane equations, it was possible to solve it numerically for large and small strains. The integral equation was discretized using Simpson's rule and the resulting system of equations was solved using the nonlinear Gauss-Seidel method. Error due to truncation did not prove to be a problem. The error due to roundoff was checked by double precision and it did not appear to be of any significance.

The numerical results confirmed the analytic theory. A parameter, $k=\sigma_{x}(L) / E$, arose naturally in the problem. In a sense it represents in the linear axial stress (recall (1.15)). In the linear theories, the axial stress is constant throughout the cylinder. In Fig. 1.2, the axial stress, as defined by (1.15), is evaluated at the center and plotted as a function of $k$ for a cylinder with a ratio of 0.1001 . Included is the value predicted by the limit theory of Sec. 4 and the Bromberg-Stoker theory. As can be seen, the agreement of the linear theory is only for rather small values of the axial stress. The limit theory does seem to indicate the behavior as the stress increases. It may be shown from the algebraic equation for $\varepsilon_{x}$ in terms of $\varepsilon_{\theta}$ that

$$
\varepsilon_{x}(L)=\left(1-v^{2}\right) k .
$$

Thus, Fig. 1.1 depicts the relationship between stresses as the axial strain is increased.

In Fig. 1.2 the displacements for the exact theory, the Bromberg-Stoker theory, and the limiting contraction at the center are plotted as functions of $k$. The cylinder ratio is about 0.1 . For the radial displacement, the Bromberg-Stoker theory is excellent throughout. The limiting contraction, as predicted in Sec. 4, is approached as $k$ increases. For the axial displacement, the prediction of Sec. 4 remains valid as $k$ increases. However, the Bromberg-Stoker theory and the exact theory diverge, showing that the BrombergStoker theory is a small-strain theory. 


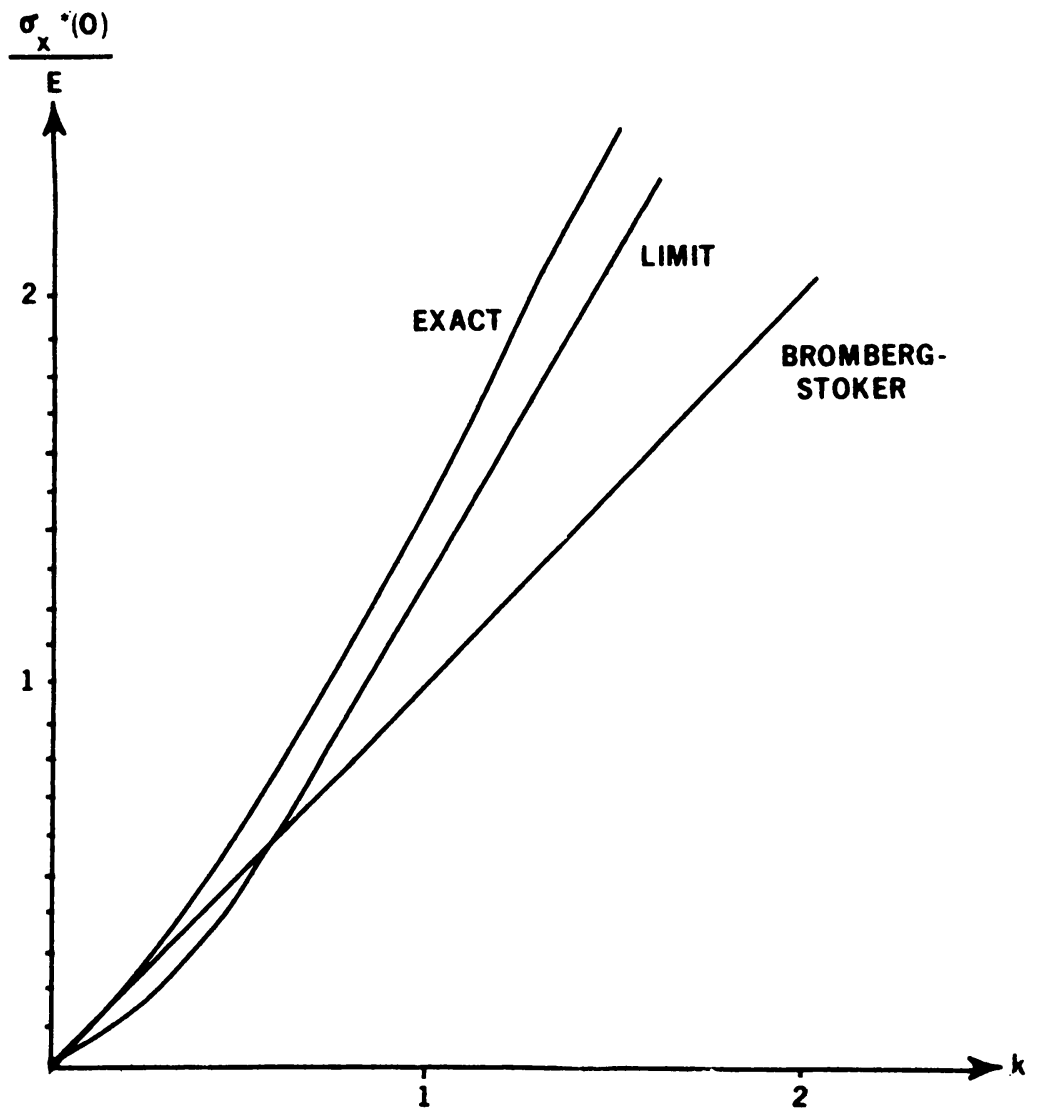

Fig. 1.1. Axial stress at the center as a function of the axial strain.
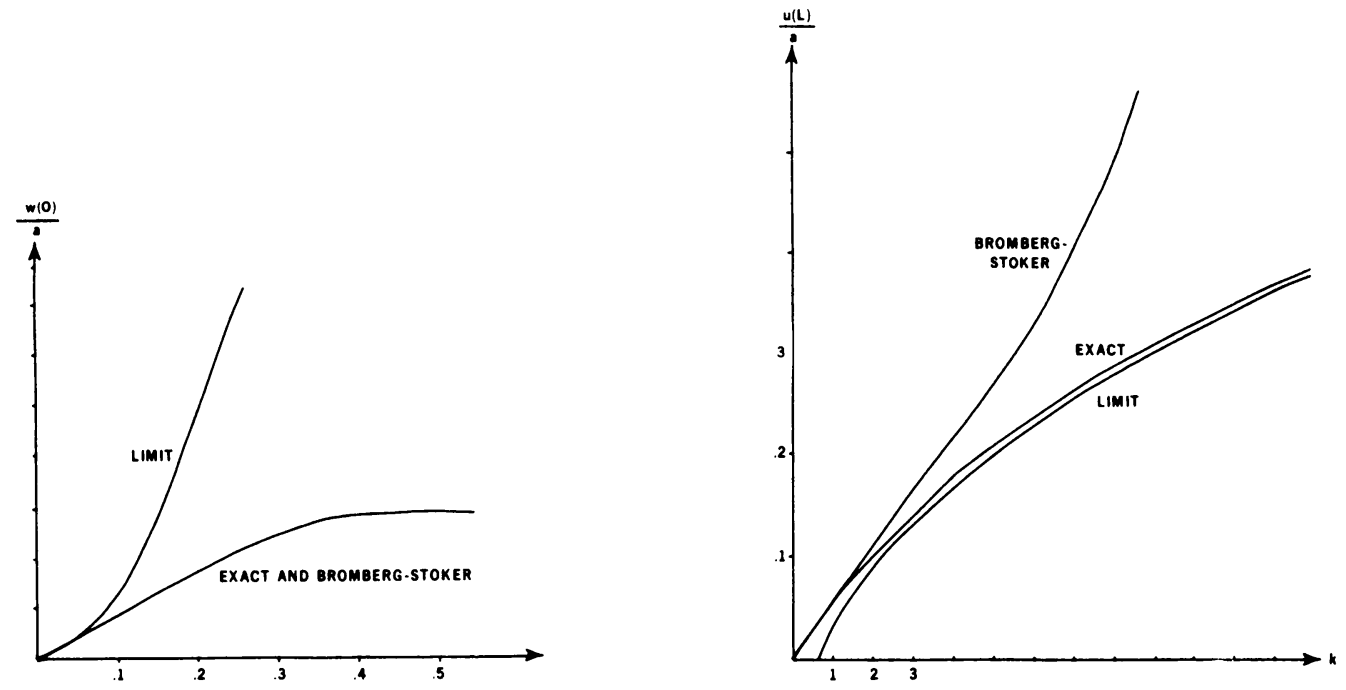

Fig. 1.2. Center contraction and axial end displacement as functions of the axial strain parameter. The ratio cylinder length : radius is 0.1 . 
The equations were also run holding $k$ fixed and allowing the cylinder ratio $B$ to vary. When $k$ was chosen small, i.e. $k=0.01$, the displacements of the exact theory and the Bromberg-Stoker theory virtually coincided. Except for very stubby cylinders, the limit theory is not applicable. Fig. 1.3 shows the results for larger axial strain, i.e. $k=10$; the agreement of the limit theory and the exact was quite good.

It was apparent from the data studied that the Bromberg-Stoker theory was excellent for small strains. For larger strains, the departure is noticed first for the quantities such as stress and axial displacement (Figs. 1.1 and 1.3b). These functions are determined, in the approximate theories, from appropriate simplifications of the exact algebraic formulae. Considering the close agreement for radial displacement and radial strain, had this step been bypassed, closer agreement could have been achieved for all functions occurring in the model.
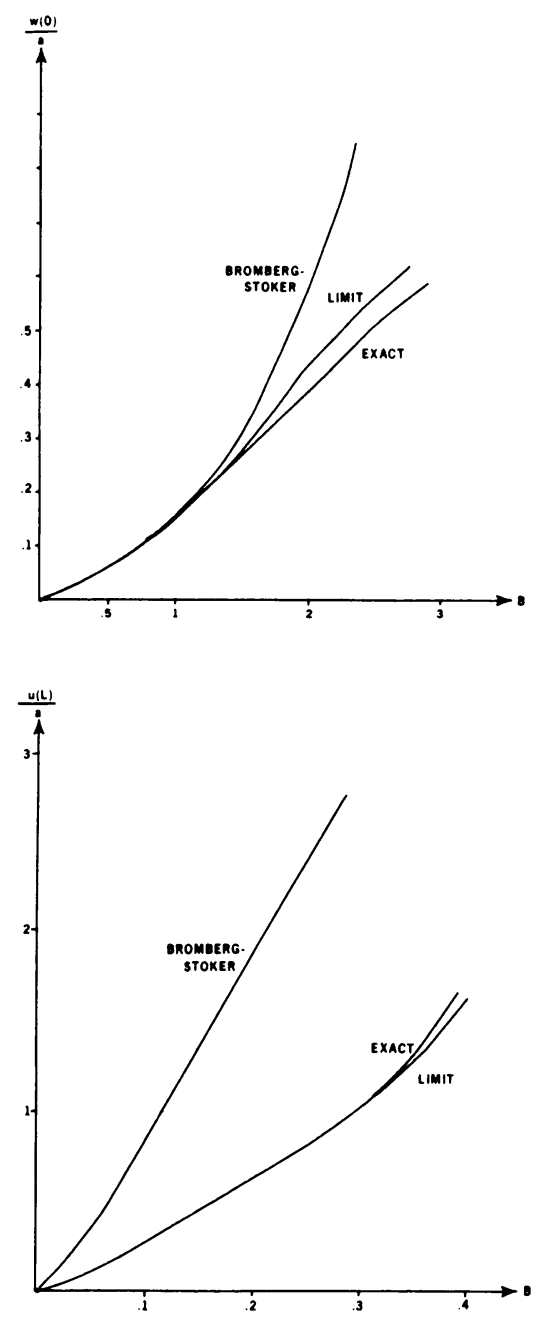

Fig. 1.3. Center contraction and axial end displacement as a function of the cylinder ratio length : diameter. The axial strain parameter $k$ is 10 . 


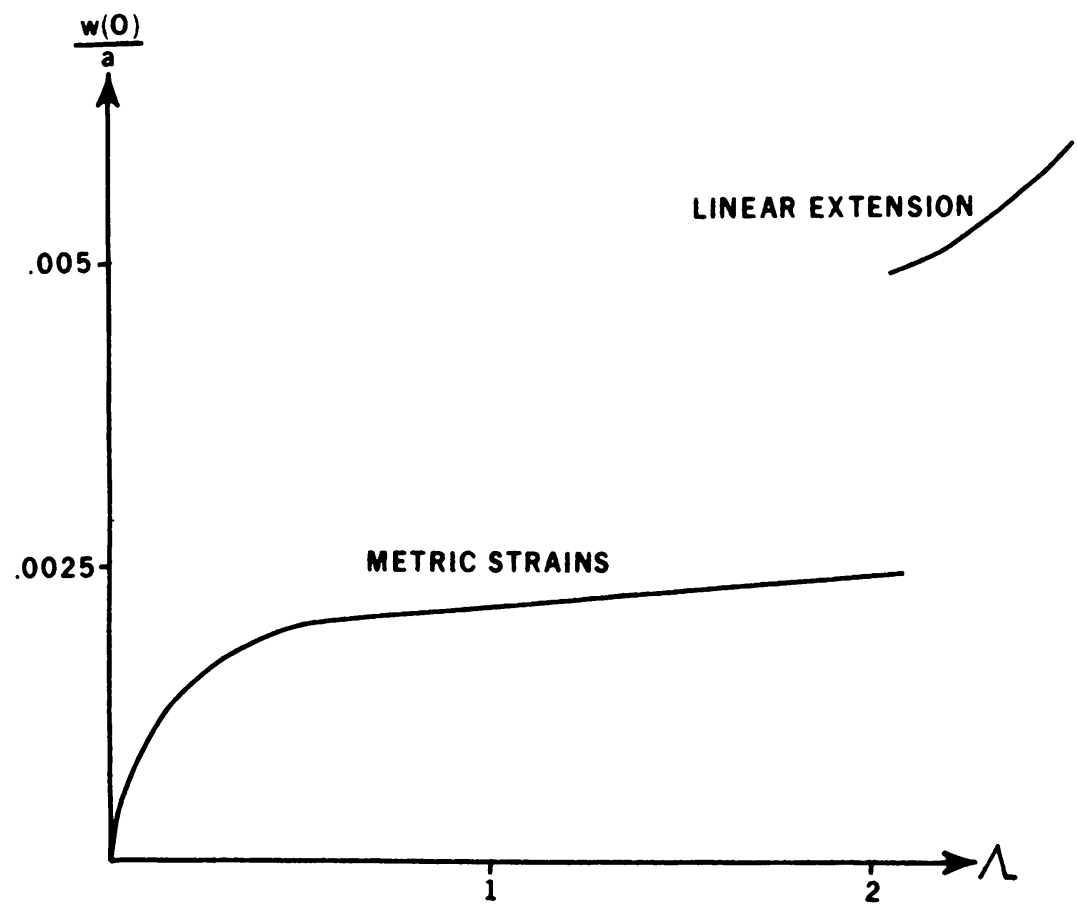

FIG. 1.4. Comparison of results using the metric strain and the linear strain.

6. Comparison with the theory using linear extensions. The strain energy function $V_{1}$, defined by (1.2), was altered in order to compare the results with those obtained in [15]. This change affected only the combination of the elastic constants in the equations corresponding to (1.3)-(1.12). If the perturbation scheme used for the integral equation is formally applied to the equations found in that paper, the solution of the perturbed equations has the same behavior. Fig. 1.4 shows the results of the attempt to compare the two theories numerically. As remarked earlier, a different definition of strains is used, i.e. (1.13) and (1.14). The discrepancy seen in Fig. 1.4 would indicate that when the strains are large, the two theories describe different materials. Therefore, any comparison in this range is not meaningful. Moreover, the numerical difficulties in that work do not make comparison possible for small strains.

\section{REFERENCES}

[1] B. Bowman, Stretching the circular cylindrical elastic sheet, Ph.D. Thesis, Math. Dept., University of Wisconsin (1979)

[2] E. Bromberg and J. J. Stoker, Nonlinear theory of curved elastic sheets, Quart. Appl. Math. 3 (1945)

[3] A. J. Callegari, H. B. Keller and E. L. Reiss, Membrane buckling: A study of solution multiplicity, Communications on Pure and Appl. Math. 24 (1971)

[4] A. J. Callegari and E. L. Reiss, Nonlinear boundary value problems for the circular membrane, Arch. for Rational Mech. and Anal. 31 (1960)

[5] A. H. Corneliussen and R. T. Shield, Finite deformation of elastic membranes with application to the stability of an inflated and extended tube, Arch. for Rational Mech. and Anal. 7 (1961)

[6] R. Courant and D. Hilbert, Methods of mathematical physics, 1, Intersc. Publ. Inc., New York (1953)

[7] T. V. Davies and E. M. James, Nonlinear differential equations, Addison, Wesley Publishing Co. (1966) 
[8] R. W. Dickey, The plane circular elastic surface under normal pressure, Arch. for Rational Mech. and Anal. 26 (1967)

[9] B. Ecke, Z Angew. Mathematica und Mechanik, 7 (1927)

[10] A. Foppl, Vorlesungen über technische Mechanik, Bd. 5, G. Teubner, Leipzig (1907)

[11] Martin A. Goldberg, An iterative solution for rotationally symmetric nonlinear membrane problems, Inter. J. of Nonlinear Mech. 1 (1966)

[12] H. Hencky, Uber den Spannungszustand in kreisrunden Platten, Zeitung Math. Phys. 63 (1915)

[13] V. V. Novozhilov, Theory of elasticity, Pergamon Student Ed. (1961)

[14] E. Reissner, Rotationally symmetric problems in the theory of thin elastic shells, Third U.S. Nat. Con. of Appl. Mech. (1958)

[15] J. J. Stoker, Topics in nonlinear elasticity, Courant Inst. of Math. Sci. Lecture Notes (1964)

[16] F. Tricomi, Integral equations, Inter. Publ. Inc., New York (1957)

[17] Chien-Heng Wu, On certain integrable nonlinear membrane solutions, Quart. of Appl. Math. 28 (1970)

[18] Chien-Heng Wu, On the solutions of a nonlinear membrane problem, SIAM J. of Appl. Math. 18 (1970) 